\title{
Implementing the Ballot Box Method to reduce social desirability bias when researching sensitive behaviours in conservation
}

\author{
Melissa Arias $^{1}$, Amy Hinsley ${ }^{1,2}$, E.J. Milner-Gulland ${ }^{1}$
}

\begin{abstract}
${ }^{1}$ Department of Zoology, University of Oxford
Oxford-Martin Programme on the Illegal Wildlife Trade

Interdisciplinary Centre for Conservation Science (ICCS)

${ }^{2}$ Wildlife Conservation Research Unit (WildCru)
\end{abstract}

Corresponding author: melissa.arias@zoo.ox.ac.uk/melissaariasg@gmail.com

\section{Introduction:}

Humans have large direct and indirect impacts on wildlife, with over-exploitation being one of the main drivers of species endangerment (Maxwell et al., 2016). As wildlife population numbers go down, there is an increasing awareness that saving species requires understanding human behaviour and motivations, beyond the scope of the natural sciences (Mascia et al., 2003). Though not without challenges, the social sciences are growing within the field of conservation (Bennett et al., 2017b), and more conservation social scientists are going to remote areas to explore human interactions with wildlife. However, in the cases where those interactions are socially perceived as negative, controversial, or illegal, even trained social scientists can find it difficult to uncover people's real attitudes and behaviours towards wildlife through direct questioning methods like surveys or interviews (Nuno and St. John, 2015). This is because respondents have a natural tendency to protect themselves from any legal consequences or social stigma associated with their actions, while also seeking to please the interviewer by providing socially acceptable answers rather than the truth, particularly when there are unequal relationships between the two parties (Grimm, 2010; Turnbull, 1944; Warner, 1965). Social desirability bias reduces the validity of direct questioning (Fisher, 1993), and may give conservation researchers a false or incomplete picture of the threats to wildlife.

A wide range of specialized indirect questioning techniques, developed by other social science disciplines have recently been used in conservation to minimize the effects of social desirability bias and participant non-response and to reach a more accurate understanding of people's relationships with wildlife (Nuno and St. John, 2015). Among these, perhaps the most popular are the Randomized Response Technique (RRT) and the Unmatched Count Technique (UCT). These methods provide additional confidentiality to respondents by eliminating their risk of identification by the interviewer, either by introducing random noise to participants' responses in the former, or by randomly blocking respondents into treatment and control groups in the case of the latter. While these methods, and others, have achieved more 'honest' responses from respondents than direct questioning in several 
conservation case studies (e.g. Davis et al., 2019; Razafimanahaka et al., 2012), researchers have warned that they should not be considered a silver bullet for research on sensitive behaviours in conservation (Hinsley et al., 2018; Nuno and St. John, 2015). Some of the limitations of these methods include that they: do not perform very well when the studied behaviour is very rare; have high levels of imprecision; require large sample sizes that challenge their cost-effectiveness; can involve complex analyses; are limited in the form and number of questions that can be asked; are rarely validated against real data on behaviours; and can intellectually burden respondents (particularly those with low education levels) without necessarily reducing their mistrust (Bova et al., 2018; Hinsley et al., 2018; Nuno and St. John, 2015). Given these challenges, some researchers are starting to advocate for simpler methods to be used to estimate sensitive behaviours in conservation, such as the Bean Method (Jones et al., 2020) and the Ballot Box Method (Bova et al., 2018).

The Ballot Box Method (BBM), also named "Ballot Box Technique", "Secret Ballot Technique" or "Informal Confidential Voting Interviews", is based on the principle of secret voting, which was officially adopted by several countries in the $19^{\text {th }}$ century to reduce voter intimidation in selecting government officials (Fredman, 2008). As with government elections, the BBM requires respondents to provide their answers to secret or sensitive questions through an anonymous ballot, placing their response inside a sealed ballot box that contains the responses from all survey respondents, without the interviewer seeing the answer. The BBM has been used to research public opinions and prevalence of sensitive issues on several topics. Some examples include voting preferences in the U.S. (Benson, 1941), the frequency of induced abortion in Brazil (e.g. Anselmo Olinto and De Carvalho Moreira Filho, 2004; Diniz et al., 2017; Diniz and Medeiros, 2010), Colombia (Zamudio et al., 1999) and México (Lara et al., 2004), the prevalence of sexually transmitted diseases in sub-Saharan Africa (Gregson et al., 2004, 2002) and non-marital sexual behaviours of men in Bangladesh (Chowdhury et al., 2012). The BBM has also been used in the field of environmental economics to test the appropriateness of contingent valuation surveys (Carson et al., 2005) and to determine people's willingness to pay for natural resources or ecosystem services in the US (Leggett et al., 2003) and the Philippines (Francisco, 2015). To the best of our knowledge, the BBM has only recently made its way into the field of wildlife conservation, through studies on bushmeat hunting in the Serengeti (Nuno, 2013), rule noncompliance among anglers in South Africa's marine-based shore fishery (Bova et al., 2018), and illegal jaguar trade in Bolivia (Arias et al., 2020). Although the BBM is at its early stages of implementation in conservation, and there have not yet been any studies validating its effectiveness against known behaviours (in conservation or in other fields, as far as we know), it is part of a growing toolbox that promises to enhance our understanding of human behaviours towards wildlife (Cerri et al., 2020). Here, we provide detailed guidance for the design and implementation of the BBM in conservation, based on a review of studies using the method and practical experience in its implementation (Box 1 ).

\section{Advantages and disadvantages of using the BBM}

The appropriateness of any given sensitive questioning technique depends on the specific behaviour of interest and the socio-cultural context of the study. Researchers interested in using these tools must consider their different benefits and drawbacks (Cerri et al., 2020; Nuno and St. John, 2015), and ideally test their performance in their own study area. The BBM offers a wide range of benefits, particularly for studies in areas with low literacy levels or with sample size constraints, but it also has disadvantages, which must be taken into account (Table 1). 
Table 1: Advantages and disadvantages of using the BBM

\begin{tabular}{|c|c|}
\hline Advantages of the BBM & Disadvantages of the BBM \\
\hline $\begin{array}{l}\text { 1. Low participant burden and minimum } \\
\text { literacy requirements make it suitable for a } \\
\text { wide range of respondents and socioeconomic } \\
\text { groups. } \\
\text { 2. Easy to understand, due to familiarity of } \\
\text { participants with anonymous voting across } \\
\text { geographies (particularly in democratic states). } \\
\text { 3. Flexibility in the number and type of } \\
\text { questions that it can support. } \\
\text { 4. It can be applied alongside other direct or } \\
\text { indirect questioning methods. } \\
\text { 5. Requires smaller sample sizes than other } \\
\text { indirect questioning techniques as it does not } \\
\text { discard data due to experimental design } \\
\text { (treatment vs. control groups) or due to the } \\
\text { introduction of random noise. } \\
6 \text {. Cheap materials make it cost-effective. } \\
\text { 7. Straightforward, non-probabilistic analysis } \\
\text { of prevalence. } \\
\text { 8. Allows for multivariate analysis of } \\
\text { behavioural drivers at the individual level. }\end{array}$ & $\begin{array}{l}\text { 1. The effectiveness of the method relies on the } \\
\text { features of the box and trust in the word of the } \\
\text { researchers that the ballot is truly anonymous. } \\
\text { 2. Its similarity to governmental elections can } \\
\text { be counterproductive if elections are } \\
\text { upcoming, if the study area has a history of } \\
\text { corruption or violence linked to elections, or if } \\
\text { democratic elections are not part of the } \\
\text { country's political tradition. } \\
\text { 3. Ethical implications from the risks of } \\
\text { participant identification must be mitigated. } \\
\text { 4. Its inclusiveness may be limited for } \\
\text { participants with extremely low or now literacy } \\
\text { levels, physical or other impairments or for } \\
\text { those who are carrying out other activities } \\
\text { simultaneously. } \\
\text { 5. Requires a confidential, quiet setting or } \\
\text { participant isolation. } \\
6 \text {. Challenges to building a safe box, as } \\
\text { appropriate materials and carpentry skills may } \\
\text { be hard to find. } \\
\text { 7. Negative or unwanted reactions to the box, } \\
\text { including curiosity or fear of what might be } \\
\text { inside. } \\
\text { 8. Inconvenience to surveyors who have to } \\
\text { carry the box around, especially in challenging } \\
\text { terrains or long distances. }\end{array}$ \\
\hline
\end{tabular}

\section{Planning and designing the BBM}

One of the key attributes of the BBM is its simplicity, but like all other sensitive questioning techniques, it requires careful consideration of each step in the planning and design process (Figure 1).

\subsection{Determine the suitability of using the BBM}

The first step in the process is for researchers to determine whether the BBM is suitable for their study context, considering how its advantages and disadvantages would play out for the particular behaviour of interest and socio-cultural context of their study. 


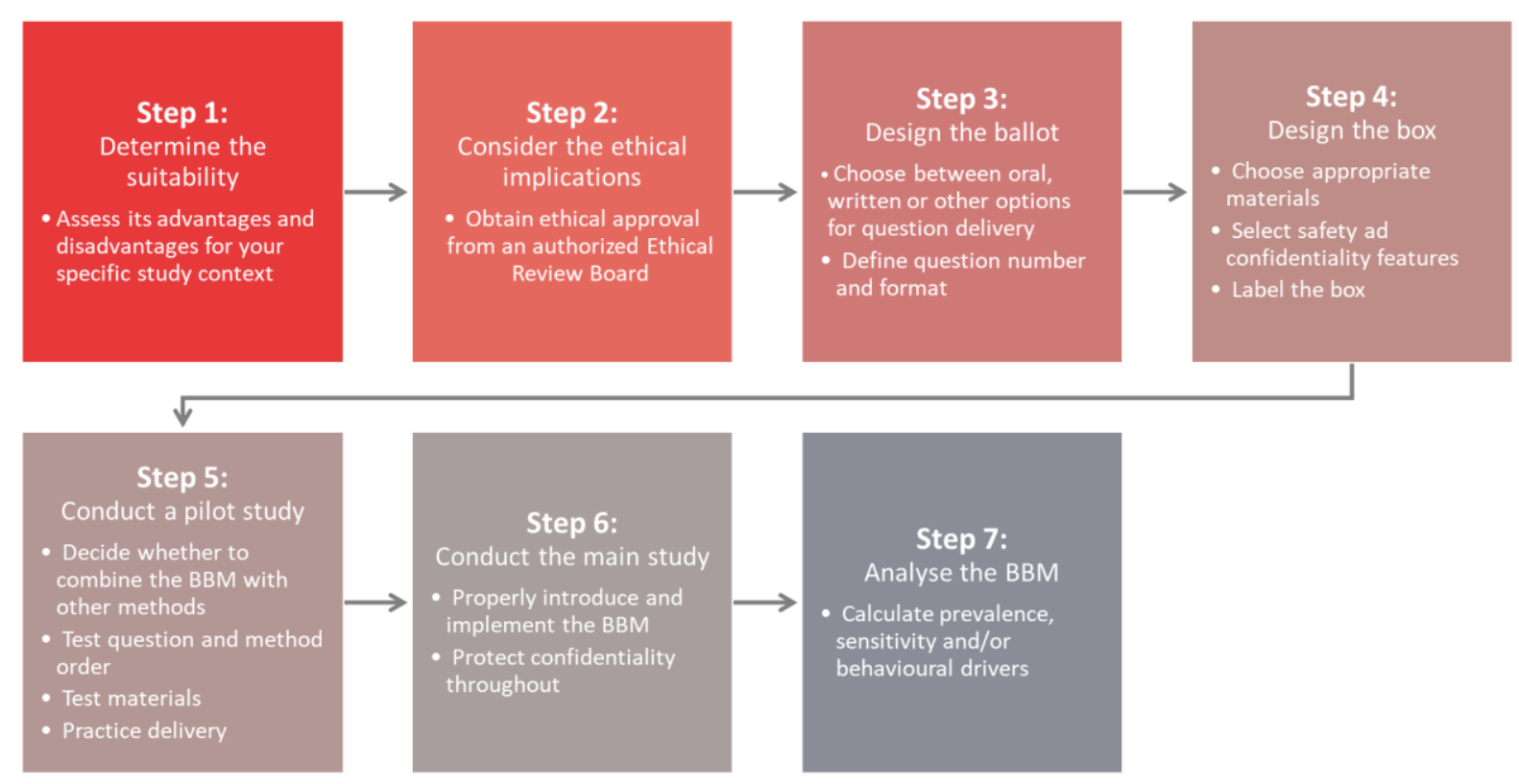

Figure 1: Flow diagram of steps for designing and implementing the BBM

\subsection{Consider the ethical implications of using the BBM}

Regardless of the field, institution or topic, researchers have an obligation to reflect on the ethical implications of their work, and to protect research participants from any adverse consequences from their involvement in the research (Brittain et al., 2020). This should involve the review of research protocols by an ethical review board, to ensure adherence to certain principles such as informed consent, voluntary participation, right to withdraw, no harm to participants, no use of deception, preservation of anonymity and confidentiality, data protection, among others (Vanclay et al., 2013). This is particularly important for research about sensitive, stigmatizing or illegal behaviour that may have social or legal consequences. Interviewees must be well aware how the study minimizes any risks to their anonymity and privacy, including the confidentiality mechanisms provided by the use of specialized questioning techniques, and of any remaining risks and the potential implications of their participation in these studies.

The BBM can offer varying levels of confidentiality depending on how it is implemented. One of the unique features of the BBM is that it is possible to include an identification code or serial number on the ballot, which allows the sensitive questions in the ballot to be re-joined with other non-sensitive and anonymous survey responses provided by the same participant (e.g. Bova et al., 2018; Chowdhury et al., 2012; Diniz and Medeiros, 2010; Madeiro and Rufino, 2012). The possibility to link the ballot to a more detailed survey allows for a rich analysis that connects reported behaviours to participant's socioeconomic characteristics or to a wide range of other potential questions on people's attitudes, perceptions, beliefs and interactions with wildlife.

However, adding an identification code to the ballot comes with ethical implications that must be considered by the researchers. Making the ballot identifiable could have negative consequences for respondents when the rest of the survey is not fully anonymous or has identifiable questions that 
make it possible to trace the answers back to the individual, or when the sample size is small such that individuals can be recognized based on their answers. In these and in all cases, researchers must take extra care to ensure that their research will do no harm to participants. This includes ensuring that their research protocols have been reviewed by authorized ethical review boards, that relevant permissions have been obtained from local communities, that surveyors are fully trained in ethical handling of sensitive information and personal data (including what to do in case of a confidentiality breach), that data management procedures are in place to ensure secure and anonymised data storage, and that participants are aware of the risks of their participation and are able to grant fully informed consent. In fairness to the participant, the identification code should be described before they grant their consent to participate in the survey, and it should be made visible in the ballot such that participants can ask more questions about it if they feel inclined to do so. Another option is to add a pre-printed encrypted code onto the ballot, that is unknown to the researchers, but which allows for the link to occur at the time of the analysis (Medeiros and Diniz, 2012).

If researchers cannot guarantee the safety of their respondents, or if they prefer to avoid the use of a ballot identification code, then they can choose to do so (Anselmo Olinto and De Carvalho Moreira Filho, 2004; Zamudio et al., 1999). This increases the confidentiality of the BBM, making it even harder to identify participants (if their answers are mixed with others). However, this sacrifices the possibility to link the behaviour to any explanatory variables at the individual level, unless those are also written into the ballot. In choosing this option, researchers can assign a number to each ballot box used in the study, to keep track of the surveyor, study area, or study group that each ballot box is associated to (Anselmo Olinto and De Carvalho Moreira Filho, 2004). This allows for between-group comparisons of the prevalence of the behaviour.

\subsection{Designing the ballot:}

The ballot is a simple piece of paper on which respondents provide their answers to sensitive questions. However, the complexity of the ballot depends on the research design. There are multiple considerations when choosing the contents of the ballot.

The first is whether to write the sensitive questions physically onto the ballot, or to have the interviewer read them aloud. Writing the questions on the ballot increases participant burden by requiring literacy skills. This can limit the study's sample size by reducing the number of people eligible for interviewing, and lead to exclusion of certain groups. It can also compromise the ballot's perceived confidentiality, since the lack of written text on the ballot provides an additional assurance to respondents that if the ballot gets misplaced or if there is a breach in the project's confidentiality, there will be no physical evidence linking the answers to the questions or to the respondent (Bova et al., 2018). However, there are also a few drawbacks to delivering the questions orally. Oral questions are less suitable for self-administration, and rely on quiet and empty surroundings. They may also lead to confusion about the order of the questions and their responses if participants are not given enough time to answer. Even stating a sensitive question orally can lead to embarrassment and resistance, and can prompt concealment of answers. It can also cause respondents to give away their answer inadvertently. Oral questions also exclude participants with hearing impairments. To minimize these issues, some studies have used alternative ways to communicate the questions to participants, such as using audio recorders and headphones (Chowdhury et al., 2012). These may not be suitable when target populations have no prior familiarity with this technology or when carrying these devices 
reveals stark wealth imbalances between researchers and participants. The same considerations apply with the use of tablets or other electronic devices.

The second consideration has to do with the number and type of questions and answers that can be added to the ballot. One of the advantages of the BBM is that multiple numbered questions can be asked using a single ballot, and that questions can take flexible forms. The simplest type of question is one that results in a binary "yes" or "no" answer (Figure 2). Depending on the respondents' literacy levels, they can be asked to write or circle the words "yes" or "no" for each numbered question based on their response, or to simply tick or check a box next to the words. Potential modifications to make the ballot even more accessible could be to add emoticons or symbols instead of words, such as thumbs up or down, although these have not been previously tested and would require piloting. In addition to the answer choices "yes" and "no", it is also possible to add a third option giving participants the opportunity to state that they "prefer not to respond", that they have "no opinion", that they are "undecided" or that they "don't know" the answer to a given question. In certain contexts, adding this third option can increase the accuracy of the study by allowing researchers to distinguish a true "yes" or "no" from other confounding reasons for selecting either answer. However, this option is not usually recommended, particularly when all respondents are in a position to state their preference, no matter how weak, between a binary "yes" or "no" answer (e.g. the question does not require specific knowledge that the respondent may not possess - Krosnick, 2002). This is because these alternative answers can introduce additional biases from respondents being embarrassed, misinterpreting the question, or lacking the willingness to undertake the cognitive effort of reflecting on their response (Krosnick, 2002). Additionally, unless some specific analysis is intended for these alternative responses, they might need to be turned into missing observations to facilitate posterior analysis, reducing the study's sample size. Piloting the questions would determine whether such nonbinary response options should be included in cases where they are selected by a meaningful proportion of the sample. An alternative way to get a sense of respondents' certainty in the answers is to ask them to indicate how sure they were about their vote through a ranking system, selecting a minimum threshold for accepting responses as valid (Francisco, 2015).

Other question types are also possible through the BBM. Numerical questions can be asked, in which case respondents can directly write the number of times they conducted a behaviour, or select from a set of given choices (Chowdhury et al., 2012). Multiple choice, Likert-scale or semi-structured questions can also be used. In fact, multiple researchers have combined longer self-administered questionnaires with the BBM by asking respondents to insert a full length questionnaire in an envelope before sliding it inside the ballot box (Lara et al., 2004; Leggett et al., 2003; Zamudio et al., 1999). Despite this great flexibility of the BBM, it is recommended to avoid designing excessively long ballots that combine sensitive and non-sensitive questions or ballots with complex instructions for answering, such as skipping or jumping to other questions, as this compromises the simplicity and low participant burden that are characteristic of the method (Medeiros and Diniz, 2012). Writing the instructions for the method on the ballot itself is also not recommended for in-person interviews because they can confuse respondents or push them to asking questions to the interviewers, increasing the risks of disclosing their answers (Medeiros and Diniz, 2012). 


\subsection{Designing the box:}

The construction of the ballot box should be considered at the early stages of the research, as depending on the location of the study, it may be difficult to find the right materials and carpentry skills to build a safe box. It is important for the box to look, and to actually be, as secure as possible, as it may otherwise undermine the intended confidentiality of the method. There are several possible ballot box materials and designs, but ideally, they should all emphasise safety while at the same time being light and comfortable enough to transport to surveying locations. Some researchers have built wooden boxes that are fully opaque (Bova et al., 2018; Chowdhury et al., 2012), while others have used acrylic to enable visibility inside the box with the goal of assuring participants that their responses are mixed with those of others (Anselmo Olinto and De Carvalho Moreira Filho, 2004).

In most cases, the ballot box has been closed with a safety lock, and surveyors explain to respondents that the key is held only by the study's lead researcher or coordinator (Anselmo Olinto and De Carvalho Moreira Filho, 2004; Bova et al., 2018). This requires building trust in the interviewer and the study as a whole. Another possibility that creates an even stronger perception of confidentiality is to build a completely sealed box (properly nailed or pasted together) that can only be opened by destroying the box (Figure 1). In any case, participants may still be suspicious about their protection through the BBM, so it is important for interviewers to ensure that any concerns are addressed and that participants are given the option to withdraw from the study at any time in case there are remaining trust issues. Some studies have also recommended labelling the box with the researchers' institutional affiliation (Bova et al., 2018) to increase the 'official' appearance of the box, and remind participants of the study's educational or research purpose.

\begin{tabular}{|c|ll|ll|}
\multicolumn{9}{|c|}{ Basic Ballot } \\
\hline \multicolumn{3}{|c|}{ Code:\#\#\# } \\
\hline 1. & YES & $\square$ & NO & $\square$ \\
\hline $\mathbf{2}$. & YES & $\square$ & NO & $\square$ \\
\hline 3. & YES & $\square$ & NO & \\
\hline 4. & YES & $\square$ & NO & \\
\hline $\mathbf{5 .}$ & YES & $\square$ & NO & \\
\hline
\end{tabular}

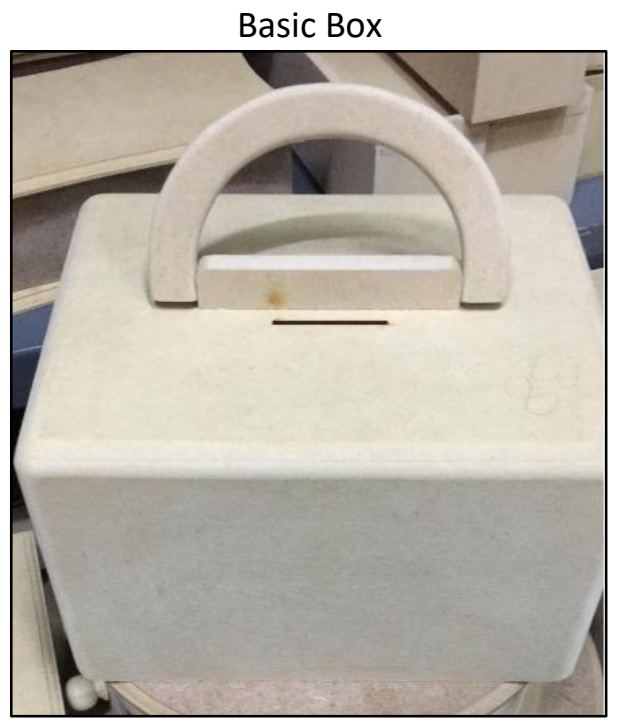

Figure 2. Example of Simple Ballot and Box (adapted from Arias et al., 2020). 


\section{Implementing the BBM}

\subsection{Piloting the BBM}

Whether the BBM is used independently or together with a larger questionnaire or with other direct or indirect questioning approaches, researchers must take the time to properly pilot their BBM surveys. Piloting is particularly crucial in mixed-method approaches, not only to guarantee the flow of the interview as a whole, but also to avoid conditioning participants to provide a given answer based on the previous questions. Introducing the BBM at the right time in the survey, by piloting the order of the methods, can also help to avoid making participants uncomfortable by asking sensitive questions before a minimum level of trust has been established with the interviewer, and to reduce bias. Piloting is also useful to ensure that the language used in the questions is culturally appropriate and that it does not lead to misinterpretations (Medeiros and Diniz, 2012). It also benefits interviewers by allowing them to practice their delivery of the BBM instructions to participants, ensuring that the procedure is clearly understood. Piloting also allows testing the ballot box materials, to ensure that they are well received by participants, and that they are comfortable, secure and strong enough to last through the entire duration of the study. Finally, like with other indirect questioning methods, in some contexts the BBM may not decrease social desirability bias or make participants feel comfortable or safe enough to provide their truthful answers. Adding a few questions to elicit participants' understanding, degree of confidence and comfort (Nuno, 2013) with the BBM and any other methods used in the survey, and obtaining surveyors' impressions on whether people understood and felt comfortable with the methods at the time of the pilot can help to identify this before resources are spent in the full sample.

\subsection{Applying the BBM jointly with other questioning methods}

As mentioned above, it is common for the BBM to be used as part of a larger survey (with or without a linking identification code), and/or together with other direct or indirect questioning approaches. This may be desirable in order to test the effectiveness of the BBM compared to direct questioning or other methods, when the sensitivity of the studied issue is unknown in a given study area. Comparing the resulting prevalence of behaviours under different questioning approaches either though a pilot study, or through a full methodological study, can reveal important insights about the prevalence and sensitivity of the behaviour of interest (and therefore the required sample size), the potential biases associated with a method in a given context (e.g. social desirability or cognitive demand), and the practicality of implementing a method at larger scales (e.g. cost, comfort). This comparison is one way to 'validate' the choice of questioning method, which is particularly important when there is no prior information on the known prevalence of the behaviour.

If this is done, the same respondent can be subjected to two or more questioning methods; or the interviewer can randomly choose which method will be applied to each respondent, alternate between the methods, split the sample into two groups, or choose some other systematic method of allocating the methods to respondents (e.g. Anselmo Olinto and De Carvalho Moreira Filho, 2004; Bova et al., 2018; Chowdhury et al., 2012; Francisco, 2015; Lara Jennifer Strickler et al., 2004). In the former scenario, the researcher must choose between increasing participant burden by asking the same questions twice with different methods, potentially affecting their response to one of the methods, or requiring a larger sample size so that the two methods can be applied to different people 
under the latter option. This choice may depend on the availability of time and resources to conduct the survey, among other factors. It also has implications for the analysis, since in the latter option, it will be necessary to either match respondents before the survey so that they are comparable, or to account for their socioeconomic differences and other confounding variables at the time of the analysis. Note that if the BBM and direct questioning are applied to the same person, and if the ballot has an identification code, it is possible to then determine the sensitivity of the issue and the reasons why some people might find it more sensitive than others, by investigating the characteristics of those who responded differently to both methods.

\subsection{Introducing the BBM to respondents}

There are a few steps that surveyors must take when introducing the BBM to respondents. First, surveyors must follow their institutional ethical protocols; beyond properly explaining their affiliation and the goals of the study to participants, they should also mention that they have taken the necessary precautions to minimize the risks associated with participating in the study involving the BBM, and that the researchers have no links to law enforcement (Bova et al., 2018). Second, surveyors should explain the rationale for using the BBM to ensure participant confidentiality. Whether or not the ballot contains an identification code, surveyors must emphasize the anonymity and non-identifiability of participants and be explicit about any risks from participating in the study. Making a reference to voting in the context of government elections can help participants to visualize and understand the method (Medeiros and Diniz, 2012), except if the country has had a history of corruption around voting in the elections, in which case this association may be counterproductive.

Third, respondents should be made aware of the exact mechanisms by which the ballot box secures their privacy and confidentiality. This includes fully explaining the contents of the ballot and providing instructions for filling it in, folding it, and depositing it inside the box. It can be useful for the surveyors to quickly fill in a mock ballot themselves to show participants how it should be done. Surveyors should also explain whether the questions will be read aloud, audio played, or whether they are written in the ballot for self-administration. If read aloud, respondents should know where and in which position the surveyor will stand in relation to them to ensure confidentiality (e.g. one meter apart and facing the other way or using some physical barrier between the two). Participants should also be aware of how much time they will have to provide their answer in between each question, and that they are welcome to ask for more time or to ask questions to the interviewer. Interviewers should also describe the safety features of the box, including details about when, how and who will open the box. It is also necessary to mention that the respondent's ballot will be mixed with the ballots of all other respondents in the study area or in the study as a whole. It may be worth mentioning the study's sample size, as this can be reassuring to respondents when the sample size is high. For the assurance of the first respondents, surveyors may want to add in a few empty ballots into the box at the beginning of the survey so that the box is evidently not empty.

Before moving forward to the implementation of the BBM, and in order to preserve the confidentiality and privacy of participants, surveyors should emphasize the importance of isolating participants from those around them, and seek a private setting to conduct the survey. In doing this, surveyors must be careful to ensure that isolating participants does not put them or their families at risk, or make them feel uncomfortable, particularly if there are gender or other unequal relationships between the surveyor and the participant. If isolation is not possible, researchers should assess the risks of 
participants disclosing the information to others around them. Depending on the context and the level of comfort and trust between the respondents and those around them, the researcher could ask bystanders to sign confidentiality forms, choose alternative ways to communicate the questions to the participant such as using audio recorders and headphones (Chowdhury et al., 2012), or stop the survey if the participant's confidentiality cannot be protected.

\section{Analysing the BBM}

The straightforward and simple non-probabilistic analysis of the BBM is one of its greatest advantages when compared to other methods like the UCT or RRT. If the answer choices were "yes" and "no", a simple proportion can be enough to obtain the prevalence of the sensitive behaviour in the sample. Other question types may require other analytical approaches, both quantitative or qualitative. If other methods are used together with the BBM, it may be desirable to test whether any differences in prevalence between the methods are statistically significant. This can be done with a simple test of proportions or chi-square tests (useful to compare single questions), or alternatively, by conducting a logistic regression on the probability of getting a "yes" response, holding the binary responses as the dependent variable, against the question, the method, and their interaction as independent variables. Multivariate regression analyses can also be used where data on covariates was also collected directly through the ballot, or by means of an identification code linking the ballot to a larger survey.

\section{Box 1. Estimating the prevalence of jaguar trade in north-western Bolivia}

Since 2013, seizures of China-bound jaguar (Panthera onca) body parts, particularly teeth, have suggested that jaguars may be joining other felids, including tigers and lions, in the market for Chinese traditional medicines and collectibles (Verheij, 2019). Bolivia has become the epicentre of jaguar body part seizures linked to Chinese demand (Nunez \& Aliaga-Rossel, 2017). In the country, a suspected 200 jaguars were killed in recent years to supply this trade, leading to 21 legal processes involving Chinese and national traders (Romo, 2020).

In order to uncover the prevalence and characteristics of this illegal behaviour, Arias et al., (2020) interviewed 1107 people in a rural area implicated in jaguar trade in north-western Bolivia, using direct questioning alongside and the Ballot Box Method (BBM). The researchers chose the BBM after conducting a pilot study on a sample of 100 people in a similar area, including other sensitive questioning techniques such as the Randomized Response Technique, Crosswise Technique, Unmatched Count Technique and Nominative Technique (Nuno \& St. John, 2015). 


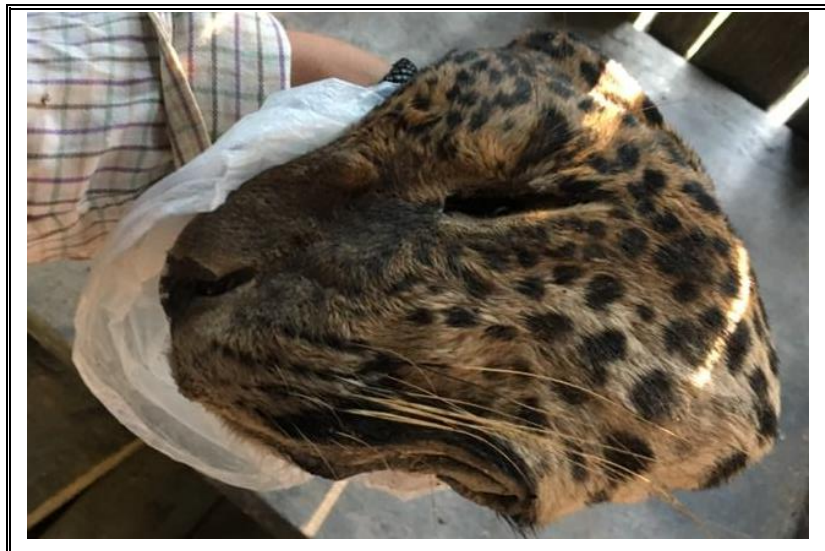

The choice of method was based on respondents' responses to questions about the method's ease of understanding, perceived confidentiality and degree of comfort (Nuno, 2013), elicited during the pilot. For the main study, the researchers asked eight yes/no questions about participants' engagement with jaguar killing or trading, using both direct questioning and the BBM, as part of a larger survey on perceptions about and interactions with jaguars.

Surveyors explained how the BBM works following protocols approved by an Ethics Review Board, and provided an example to participants by filling in a mock ballot. Participants were informed about the procedures for opening the box, and how the method protected their confidentiality. Each question was read aloud to participants, while the surveyor turned their back to the respondent. Each ballot had a unique identification code which enabled matching of BBM responses to the relevant anonymous questionnaire for analysis. No personal data were collected.

The study found that $46 \%$ of participants had engaged with jaguar killing or trading in the past 5 years. Thirty one percent of respondents owned jaguar body parts, most commonly skins, fat and teeth for decorative, medicinal, and cultural purposes. The BBM resulted in a higher prevalence of all jaguar killing and trading actions than direct questioning; averaging across actions, $6.7 \%$ of respondents admitted to actions through the BBM but not directly. However, this higher prevalence was not statistically significant, except for one action: asking others to kill a jaguar.

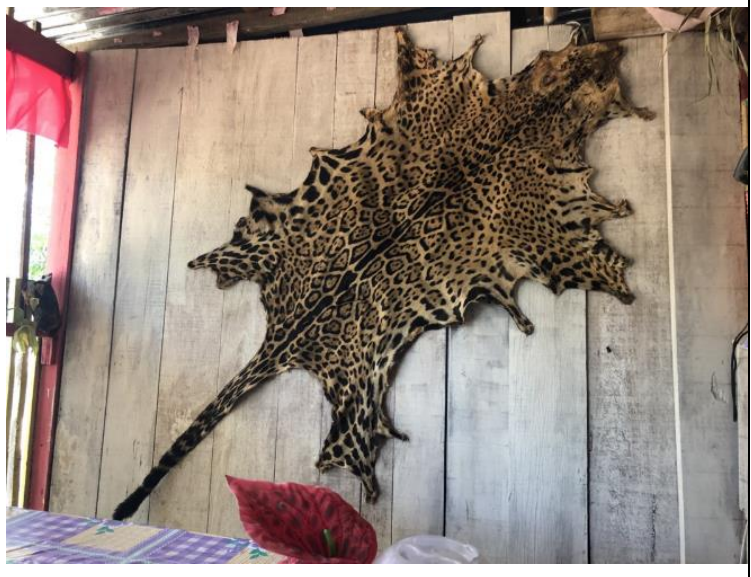

Older participants were less likely to find the questions sensitive $(\mathrm{OR} 0.99,95 \% \mathrm{Cl} 0.97-1.00$, $p<0.01)$, as were those who were not aware of the protected legal status of jaguars $(\mathrm{OR} 0.37,95 \% \mathrm{Cl}$ $0.31-0.43, p<0.001)$. A small number of respondents $(4.3 \%)$ admitted to actions in the direct question but not the BBM, suggesting they did not understand, trust or feel comfortable with the BBM.

Photo credits: Melissa Arias and Paola Nogales-Ascarrunz

\section{Conclusions}

Based on an old technology, the BBM is a relatively new indirect questioning tool to add to the conservation toolbox. Its wide range of benefits, and particularly its simplicity, make it a promising method to reduce social desirability bias in social surveys on sensitive behaviours in conservation. We provide guidance on BBM design and implementation, and encourage its wider application in conservation. At the same time, we emphasize several important considerations for applying the 
method effectively and in a way that protects participants and their confidentiality. As with any research involving humans, researchers intending to apply the BBM to study sensitive behaviours in conservation should review their research protocols with authorized ethical review boards, and pilot their surveys to ensure that the BBM is appropriate for their study's local context. While the BBM has been successful in other disciplines, further experimentation with the method will reveal its suitability and effectiveness for conservation.

\section{References}

Anselmo Olinto, M.T., De Carvalho Moreira Filho, D., 2004. Estimating the frequency of induced abortion: A comparison of two methods. Rev. Panam. Salud Publica/Pan Am. J. Public Heal. 15, 331-336. https://doi.org/10.1590/S1020-49892004000500008

Arias, M., Hinsley, A., Nogales-Ascarrunz, P., Negroes, N., Glikman, J.A., Milner-Gulland, E.J., 2020. Prevalence and characteristics of jaguar trade in north-western Bolivia. SocArXiv. https://doi.org/10.31235/OSF.IO/S4P6R

Bennett, N.J., Roth, R., Klain, S.C., Chan, K., Christie, P., Clark, D.A., Cullman, G., Curran, D., Durbin, T.J., Epstein, G., Greenberg, A., Nelson, M.P., Sandlos, J., Stedman, R., Teel, T.L., Thomas, R., Veríssimo, D., Wyborn, C., 2017a. Conservation social science: Understanding and integrating human dimensions to improve conservation. Biol. Conserv.

https://doi.org/10.1016/j.biocon.2016.10.006

Bennett, N.J., Roth, R., Klain, S.C., Chan, K.M.A., Clark, D.A., Cullman, G., Epstein, G., Nelson, M.P., Stedman, R., Teel, T.L., Thomas, R.E.W., Wyborn, C., Curran, D., Greenberg, A., Sandlos, J., Veríssimo, D., 2017b. Mainstreaming the social sciences in conservation. Conserv. Biol. 31, 5666. https://doi.org/10.1111/cobi.12788

Benson, L.E., 1941. Studies in Secret Ballot Technique. Public Opin. Q. 5, 79-82. https://doi.org/10.2307/2744879

Bova, C.S., Aswani, S., Farthing, M.W., Potts, W.M., 2018. Limitations of the random response technique and a call to implement the ballot box method for estimating recreational angler compliance using surveys. Fish. Res. 208, 34-41. https://doi.org/10.1016/J.FISHRES.2018.06.017

Brittain, S., Ibbett, H., Lange, E., Dorward, L., Hoyte, S., Marino, A., Milner-Gulland, E.J., Newth, J., Rakotonarivo, S., Veríssimo, D., Lewis, J., 2020. Ethical considerations when conservation research involves people. Conserv. Biol. 34, 925-933. https://doi.org/10.1111/cobi.13464

Carson, R.T., Hanemann, W.M., Kopp, R.J., Krosnick, J.A., Mitchell, R.C., Presser, S., Ruud, P.A., Smith, V.K.K., 2005. Was the NOAA Panel Correct about Contingent Valuation? SSRN Electron. J. https://doi.org/10.2139/ssrn.31992

Cerri, J., Davis, E., Veríssimo, D., Glikman, J.A., 2020. A fine balance: specialized questioning techniques and their use in conservation. EcoEvoRxiv. https://doi.org/10.32942/OSF.IO/F5E89

Chowdhury, M.E., Alam, N., Anwar, I., Ahmed, A., Saidel, T., Mallick, P.S., Kelly, R., Streatfield, P.K., 2012. Assessment of non-marital sexual behaviours of men in bangladesh: A methodological 
experiment using a modified confidential ballot-box method. Int. J. STD AIDS 23. https://doi.org/10.1258/ijsa.2009.009157

Davis, E.O., Crudge, B., Lim, T., O'Connor, D., Roth, V., Hunt, M., Glikman, J.A., 2019. Understanding the prevalence of bear part consumption in Cambodia: A comparison of specialised questioning techniques. PLoS One 14, e0211544. https://doi.org/10.1371/journal.pone.0211544

Diniz, D., Medeiros, M., 2010. Aborto no Brasil: Uma pesquisa domiciliar com técnica de urna. Cienc. e Saude Coletiva 15, 959-966. https://doi.org/10.1590/s1413-81232010000700002

Diniz, D., Medeiros, M., Madeiro, A., 2017. National abortion survey 2016. Cienc. e Saude Coletiva 22, 653-660. https://doi.org/10.1590/1413-81232017222.23812016

Fisher, R.J., 1993. Social Desirability Bias and the Validity of Indirect Questioning. J. Consum. Res. 20, 303. https://doi.org/10.1086/209351

Francisco, J.P.S., 2015. Willingness to pay for air quality improvements from using electric Jeepneys in metro Manila. Singapore Econ. Rev. 60. https://doi.org/10.1142/S0217590815500733

Fredman, L.E., 2008. The Introduction of the Australian Ballot in the United States. Aust. J. Polit. Hist. 13, 204-220. https://doi.org/10.1111/j.1467-8497.1967.tb00802.x

Gregson, S., Mushati, P., White, P.J., Mlilo, M., Mundandi, C., Nyamukapa, C., 2004. Informal confidential voting interview methods and temporal changes in reported sexual risk behaviour for HIV transmission in sub-Saharan Africa, in: Sexually Transmitted Infections. BMJ Publishing Group, p. ii36. https://doi.org/10.1136/sti.2004.012088

Gregson, S., Zhuwau, T., Ndlovu, J., Nyamukapa, C., 2002. Methods to Reduce Social Desirability Bias in Sex Surveys in Low Development Settings: Experience in Zimbabwe. Sex. Transm. Dis. 29, 568-575.

Grimm, P., 2010. Social Desirability Bias, in: Wiley International Encyclopedia of Marketing. John Wiley \& Sons, Ltd, Chichester, UK. https://doi.org/10.1002/9781444316568.wiem02057

Hinsley, A., Keane, A., St.John, F.A. V, Ibbett, H., Nuno, A., 2018. Asking sensitive questions using the Unmatched Count Technique: Applications and guidelines for conservation. Methods Ecol. Evol. https://doi.org/10.1111/2041-210X.13137

Jones, S., Papworth, S., Keane, A.M., Vickery, J., St John, F.A. V., 2020. The bean method as a tool to measure sensitive behaviour. Conserv. Biol. cobi.13607. https://doi.org/10.1111/cobi.13607

Krosnick, J.A., 2002. The Causes of No-Opinion Responses to Attitude Measures in Surveys: They Rarely Are What They Appear to Be., in: R. M. Groves, D.A. Dillman, J.L. Eltinge, and R.J.A.L. (eds. ). (Ed.), In Survey Nonresponse. Wiley, New York, pp. 88-100.

Lara, D., Strickler, J., Díaz Olavarrieta, C., Ellertson, C., 2004. Measuring Induced Abortion in Mexico: A Comparison of Four Methodologies. Sociol. Methods Res. 32, 529-558. https://doi.org/10.1177/0049124103262685

Leggett, C.G., Kleckner, N.S., Boyle, K.J., Duffield, J.W., Mitchell, R.C., 2003. Social desirability bias in contingent valuation surveys administered through in-person interviews. Land Econ. 79, 561575. https://doi.org/10.2307/3147300

Madeiro, A.P., Rufino, A.C., 2012. Aborto induzido entre prostitutas: Um levantamento pela técnica 
de urna em Teresina - Piauí. Cienc. e Saude Coletiva 17, 1735-1743.

https://doi.org/10.1590/S1413-81232012000700012

Mascia, M.B., Brosius, J.P., Dobson, T.A., Forbes, B.C., Horowitz, L., McKean, M.A., Turner, N.J., 2003. Conservation and the social sciences. Conserv. Biol. https://doi.org/10.1046/j.15231739.2003.01738.x

Maxwell, S.L., Fuller, R.A., Brooks, T.M., Watson, J.E.M., 2016. Biodiversity: The ravages of guns, nets and bulldozers. Nature. https://doi.org/10.1038/536143a

Medeiros, M., Diniz, D., 2012. Recomendações para inquéritos sobre aborto usando a técnica de urna. Cienc. e Saude Coletiva 17, 1721-1724. https://doi.org/10.1590/S141381232012000700010

Nuno, A., 2013. Managing social-ecological systems under uncertainty: implications for conservation. Imperial College London, London.

Nuno, A., St. John, F.A.V., 2015. How to ask sensitive questions in conservation: A review of specialized questioning techniques. Biol. Conserv. 189, 5-15. https://doi.org/10.1016/J.BIOCON.2014.09.047

Razafimanahaka, J.H., Jenkins, R.K.B., Andriafidison, D., Randrianandrianina, F., Rakotomboavonjy, V., Keane, A., Jones, J.P.G., 2012. Novel approach for quantifying illegal bushmeat consumption reveals high consumption of protected species in Madagascar. Oryx 46, 584-592. https://doi.org/10.1017/S0030605312000579

Turnbull, W., 1944. Secret vs. Nonsecret Ballots, in: Research, H.C. and R.A. in the O. of P.O. (Ed.), Gauging Public Opinion. Princeton University Press, Port Washington NT, pp. 77-97.

Vanclay, F., Baines, J.T., Taylor, C.N., 2013. Principles for ethical research involving humans: Ethical professional practice in impact assessment Part I. Impact Assess. Proj. Apprais. 31, 243-253. https://doi.org/10.1080/14615517.2013.850307

Warner, S.L., 1965. Randomized Response: A Survey Technique for Eliminating Evasive Answer Bias. J. Am. Stat. Assoc. 60, 63-69. https://doi.org/10.1080/01621459.1965.10480775

Zamudio, L., Rubiano, N., Wartenberg, L., 1999. The Incidence and Social and Demographic Characteristics of Abortion in Colombia, in: Mundigo, A.I., Indriso, C., World Health Organization (Eds.), Abortion in the Developing World. New Delhi: Vistaar, p. 498. 